KEK-TH-418

KEK preprint 94-147

KANAZAWA-94-24

November 1994

\title{
Constraints on the electroweak universal parameters and the top and Higgs masses from updated LEP/SLC data
}

\author{
S. Matsumoto \\ Theory Group, KEK, Tsukuba, Ibaraki 305, Japan \\ and \\ Department of Physics, Kanazawa University, Kanazawa, 920-11, Japan
}

\begin{abstract}
A global analysis is performed using the latest data from LEP and SLC. Constraints on the electroweak universal parameters $(S, T, U)$ and on the masses of the top quark and Higgs boson within the Standard Model (SM) are investigated. The uncertainties due to the QCD and QED effective couplings, $\alpha_{s}\left(m_{Z}\right)$ and $\bar{\alpha}\left(m_{Z}^{2}\right)$, are examined in detail. Even though the mean value of $S$ is increased to be consistent with zero, the naive Technicolor models are still disfavored due to its reduced error. Within the SM, we find the $90 \% \mathrm{CL}$ constraints; $133 \mathrm{GeV}<m_{t}<190 \mathrm{GeV}$ and $10 \mathrm{GeV}<m_{H}<440 \mathrm{GeV}$ for $\alpha_{s}\left(m_{Z}\right)=0.116$ and $1 / \bar{\alpha}\left(m_{Z}^{2}\right)=128.72$. The experimental constraints on the $Z b_{L} b_{L}$ vertex form-factor, $\bar{\delta}_{b}\left(m_{Z}^{2}\right)$, play an important role in disfavoring the region of large $m_{t}\left(m_{t} \sim 200 \mathrm{GeV}\right)$ and large $m_{H}\left(m_{H} \sim 1000 \mathrm{GeV}\right)$. If $m_{t}$ is precisely known, the present electroweak data give a rather strict upper bound on the Higgs mass, $m_{H}<140(300) \mathrm{GeV}$ at $95 \% \mathrm{CL}$, for $m_{t}=160(175) \mathrm{GeV}$ and for the above $\alpha_{s}\left(m_{Z}\right)$ and $\bar{\alpha}\left(m_{Z}^{2}\right)$.
\end{abstract}


During 1993 the four LEP experiments performed a high precision scan near the $Z$ boson resonance [1,2]. The uncertainties in the $Z$ parameters, such as the total $Z$ width and the various asymmetries, are significantly reduced from the previous results [3]. Also much improved is the measurement of the left-right polarization asymmetry at SLC [4]. Additionally, the $W$-mass measurements at Tevatron were also improved in 1993 [5]. More striking is evidence for the top quark reported by the CDF Collaboration [6]. These data may provide hints about new physics beyond the Standard Model (SM) through quantum effects prior to its discovery at future collider experiments.

Hence it is important to interpret various data in a systematic way that is convenient not only for testing the SM, but also for studying consequences of new physics. In this letter we present an update of the comprehensive study of the electroweak data based on the formalism of ref. [7]. A theoretical fit of the electroweak data has been performed by allowing the gauge boson propagator corrections and the $Z b_{L} b_{L}$ vertex form-factor to vary freely within the generic $\mathrm{SU}(2)_{\mathrm{L}} \times \mathrm{U}(1)_{\mathrm{Y}}$ gauge theory framework; SM dominance of the remaining vertex and box corrections has been assumed. The formalism allows us to obtain constraints on the universal $(S, T, U)$ parameters [8] which are modified in ref. [7] to include the SM contribution, as functions of $\alpha_{s}\left(m_{Z}\right)$, the QED effective charge, $\bar{\alpha}\left(m_{Z}^{2}\right)$, and the $Z b_{L} b_{L}$ vertex correction factor, $\bar{\delta}_{b}\left(m_{Z}^{2}\right)$, which are not precisely known at present. By neglecting new physics contributions to the parameters $S, T, U$ and $\bar{\delta}_{b}\left(m_{Z}^{2}\right)$, we obtain constraints on $m_{t}$ and $m_{H}$ as functions of $\alpha_{s}$ and $\bar{\alpha}\left(m_{Z}^{2}\right)$. All results are expressed such that consequences of future improvements in the estimate of $\alpha_{s}\left(m_{Z}\right)$ and $\bar{\alpha}\left(m_{Z}^{2}\right)$ are immediately transparent.

We start with a brief review, but for details and further references the reader is referred to ref. [7]. Then we discuss the significance of the updated data on the universal parameters of the neutral-current sector, $\left(\bar{g}_{Z}^{2}\left(m_{Z}^{2}\right), \bar{s}^{2}\left(m_{Z}^{2}\right)\right)$, which are nearly equivalent to the modified $(S, T)$ parameters of ref. [7]. Determination of the $Z b_{L} b_{L}$ vertex correction factor $\bar{\delta}_{b}\left(m_{Z}^{2}\right)$ and that of $\alpha_{s}$ from various electroweak measurements are also discussed. We also discuss the situation where all radiative effects are dominated by the SM contribution; here we place constraints on the masses of the top quark and the Higgs boson which are the only relevant free parameters of the theory. Finally, we modify this discussion to the scenario where $m_{t}$ is measured accurately.

In generic $\mathrm{SU}(2)_{\mathrm{L}} \times \mathrm{U}(1)_{\mathrm{Y}}$ theories, the universal effective form-factors that characterize the gauge boson propagator corrections are given by

$$
\begin{aligned}
\frac{1}{\bar{e}^{2}\left(q^{2}\right)} & =\frac{1}{\hat{e}^{2}(\mu)}\left[1+\operatorname{Re} \bar{\Pi}_{T, \gamma}^{\gamma \gamma}\left(q^{2}\right)\right], \\
\bar{s}^{2}\left(q^{2}\right) & =\hat{s}^{2}(\mu)+\frac{\bar{e}^{2}\left(q^{2}\right)}{\hat{e}(\mu) \hat{g}_{Z}(\mu)} \operatorname{Re} \bar{\Pi}_{T, \gamma}^{\gamma Z}\left(q^{2}\right),
\end{aligned}
$$


Table 1 Experimental data on $Z$-pole and the SM predictions.

\begin{tabular}{|c|c|c|c|c|c|}
\hline measurement & data & \multicolumn{4}{|c|}{ SM prediction $\left(\alpha_{s}=0.116, \delta_{\alpha}=0\right)$} \\
\hline$m_{t}(\mathrm{GeV})$ & & 150 & 150 & 175 & 175 \\
\hline$m_{H}(\mathrm{GeV})$ & & 100 & 1000 & 100 & 1000 \\
\hline LEP [2] & & & & & \\
\hline$m_{Z}(\mathrm{GeV})$ & $91.1888 \pm 0.0044$ & \multicolumn{4}{|c|}{ (input) } \\
\hline$\Gamma_{Z}(\mathrm{GeV})$ & $2.4974 \pm 0.0038$ & 2.4906 & 2.4823 & 2.4965 & 2.4877 \\
\hline$\sigma_{h}^{0}(\mathrm{nb})$ & $41.49 \pm 0.12$ & 41.47 & 41.48 & 41.48 & 41.49 \\
\hline$R_{\ell} \equiv \Gamma_{h} / \Gamma_{\ell}$ & $20.795 \pm 0.040$ & 20.738 & 20.714 & 20.729 & 20.706 \\
\hline$A_{\mathrm{FB}}^{0, \ell}$ & $0.0170 \pm 0.0016$ & 0.0153 & 0.0132 & 0.0167 & 0.0145 \\
\hline \multicolumn{6}{|l|}{$\tau$ polarization: } \\
\hline$A_{\tau}$ & $0.143 \pm 0.010$ & 0.142 & 0.132 & 0.148 & 0.138 \\
\hline & $0.135 \pm 0.011$ & 0.142 & 0.132 & 0.148 & 0.138 \\
\hline \multicolumn{6}{|l|}{$b$ and $c$ quark results: } \\
\hline$R_{b} \equiv \Gamma_{b} / \Gamma_{h}$ & $0.2202 \pm 0.0020$ & 0.2165 & 0.2166 & 0.2157 & 0.2157 \\
\hline$R_{c} \equiv \Gamma_{c} / \Gamma_{h}$ & $0.1583 \pm 0.0098$ & 0.1718 & 0.1717 & 0.1721 & 0.1720 \\
\hline$A_{\mathrm{FB}}^{0, b}$ & $0.0967 \pm 0.0038$ & 0.0994 & 0.0923 & 0.1038 & 0.0965 \\
\hline$A_{\mathrm{FB}}^{0, c}$ & $0.0760 \pm 0.0091$ & 0.0710 & 0.0655 & 0.0744 & 0.0688 \\
\hline \multicolumn{6}{|l|}{$\mathrm{SLC}$ 怔 } \\
\hline$A_{\mathrm{LR}}^{0}$ & $0.1637 \pm 0.0075$ & 0.1420 & 0.1320 & 0.1482 & 0.1380 \\
\hline$\chi^{2} /($ d.o.f. $)$ & & $19.8 / 11$ & $50.7 / 11$ & $17.6 / 11$ & $31.8 / 11$ \\
\hline \multicolumn{6}{|c|}{111} \\
\hline \multicolumn{6}{|c|}{$\overline{\bar{g}_{Z}^{2}\left(q^{2}\right)}=\overline{\hat{g}_{Z}^{2}(\mu)}$} \\
\hline \multicolumn{6}{|c|}{$1-1$} \\
\hline
\end{tabular}

where the hatted couplings, $\hat{e} \equiv \hat{g} \hat{s} \equiv \hat{g}_{Z} \hat{s} \hat{c}$, and all ultraviolet-singular loop functions are renormalized in the $\overline{\mathrm{MS}}$ scheme. We also use the notation $\bar{\Pi}_{T, V}^{A B}\left(q^{2}\right) \equiv\left[\bar{\Pi}_{T}^{A B}\left(q^{2}\right)-\right.$ $\left.\bar{\Pi}_{T}^{A B}\left(m_{V}^{2}\right)\right] /\left(q^{2}-m_{V}^{2}\right) ; m_{V}$ is the physical mass of the gauge boson ' $V$ ' (that is, $m_{V}=$ $m_{W}, m_{Z}$ or $m_{\gamma}$ with $\left.m_{\gamma}=0\right)$ and the subscript $T$ denotes the transverse part of the vacuum polarization tensor, $\Pi_{\mu \nu}(q)$. The 'overlines' denote inclusion of the pinch terms 9 [11]. The explicit expressions for $\bar{\Pi}$ 's in the SM are found in ref. [7]. The helicity amplitudes of neutral-current processes are expressed in terms of these charge form-factors plus appropriate vertex and box corrections. Hence the charge form-factors can be directly extracted from the experimental data by assuming SM dominance to the vertex and box corrections, and the extracted values can be compared with various theoretical predictions.

The experimental data on the $Z$-pole [2, 4] which are used in our analysis are listed in Table 1. Also shown are the SM predictions for $\left(m_{t}, m_{H}\right)=(150 \mathrm{GeV}, 100 \mathrm{GeV})$, 
Table $2 \bar{s}^{2}\left(m_{Z}^{2}\right)$ from various asymmetries. The column ' $\chi$ ' denotes the deviation from the combined mean value normalized by each error.

\begin{tabular}{|l|c|r|c|}
\hline measurements & extracted $\bar{s}^{2}\left(m_{Z}^{2}\right)$ & \multicolumn{1}{|c|}{$\chi$} & $\chi_{\min }^{2} /$ (d.o.f.) \\
\hline$A_{\mathrm{FB}}^{0, \ell}$ & $0.2302 \pm 0.0009$ & -0.5 & - \\
$A_{\tau}$ & $0.2310 \pm 0.0013$ & 0.3 & - \\
$A_{e}$ & $0.2321 \pm 0.0014$ & 1.0 & - \\
$A_{\mathrm{LR}}^{0}$ & $0.2284 \pm 0.0010$ & -2.4 & - \\
$A_{\mathrm{FB}}^{0, b}$ & $0.2316 \pm 0.0007$ & 1.5 & - \\
$A_{\mathrm{FB}}^{0, c}$ & $0.2300 \pm 0.0021$ & -0.3 & - \\
\hline$A_{\mathrm{FB}}^{0, \ell}, A_{e}, A_{\tau}, A_{\mathrm{LR}}^{0}$ & $0.2301 \pm 0.0005$ & -1.1 & $5.7 / 3$ \\
\hline$A_{\mathrm{FB}}^{0, \ell}, A_{\tau}, A_{e}, A_{\mathrm{LR}}^{0}, A_{\mathrm{FB}}^{0, b}, A_{\mathrm{FB}}^{0, c}$ & $0.2306 \pm 0.0004$ & 0 & $9.4 / 5$ \\
\hline
\end{tabular}

$(150 \mathrm{GeV}, 1000 \mathrm{GeV}),(175 \mathrm{GeV}, 100 \mathrm{GeV})$ and $(175 \mathrm{GeV}, 1000 \mathrm{GeV})$, with $\alpha_{s}\left(m_{Z}\right)=$ 0.116 [12] and $\delta_{\alpha} \equiv 1 / \bar{\alpha}\left(m_{Z}^{2}\right)-128.72=0$ [7], where $\bar{\alpha}\left(m_{Z}^{2}\right) \equiv \bar{e}^{2}\left(m_{Z}^{2}\right) / 4 \pi$. The correlations in the errors of the $Z$ line-shape parameters $\left(m_{Z}, \Gamma_{Z}, \sigma_{h}, R_{\ell}, A_{\mathrm{FB}}^{0, \ell}\right)$ and those of the measurements concerning the bottom and charm quarks $\left(R_{b}, R_{c}, A_{\mathrm{FB}}^{0, b}, A_{\mathrm{FB}}^{0, c}\right)$ as reported in ref. [目] are taken into account in the fits. The $Z$ mass, $m_{Z}=91.1888 \mathrm{GeV}$, is treated as an input parameter neglecting its error. This is justified because the experimental uncertainty and correlations are so small. In the following analysis, (a) we assume that only three neutrinos $\left(N_{\nu}=3\right)$ contribute to the invisible width of $Z$, (b) we include perturbative QCD corrections with finite quark-mass effects, (c) we calculate vertex and box corrections within the SM, (d) except for corrections due to the $Z b_{L} b_{L}$ vertex. The $Z b_{L} b_{L}$ vertex correction is represented by the form-factor $\bar{\delta}_{b}\left(m_{Z}^{2}\right)$ which, in many cases, is treated as an external parameter similar to the treatment of $\alpha_{s}\left(m_{Z}\right)$. The SM predicts $\bar{\delta}_{b}=-0.0079,-0.0100,-0.0123$ for $m_{t}=150,175,200 \mathrm{GeV}$, respectively, with $m_{H}=100 \mathrm{GeV}$ and $\alpha_{s}=0.116$; we make the abbreviations $\bar{\delta}_{b} \equiv \bar{\delta}_{b}\left(m_{Z}^{2}\right)$ and $\alpha_{s} \equiv \alpha_{s}\left(m_{Z}\right) \overline{\mathrm{MS}}$. Dependence on $m_{H}$ and $\alpha_{s}$ at the two-loop level is present but is not significant [7].

Under these conditions all $Z$ parameters are expressed in terms of $\bar{g}_{Z}^{2}\left(m_{Z}^{2}\right), \bar{s}^{2}\left(m_{Z}^{2}\right)$, $\bar{\delta}_{b}\left(m_{Z}^{2}\right)$ and $\alpha_{s}\left(m_{Z}\right)$. Among these form-factors $\bar{s}^{2}\left(m_{Z}^{2}\right)$ is determined primarily from the asymmetry measurements. Table 2 shows the fitted values of $\bar{s}^{2}\left(m_{Z}^{2}\right)$ as determined from each asymmetry measurement. The dependence of the fits on the remaining parameters, $\bar{g}_{Z}^{2}\left(m_{Z}^{2}\right), \bar{\delta}_{b}$ and $\alpha_{s}$, is negligible compared to the errors. Also shown are two combined

* From the various measurements of $\alpha_{s}\left(m_{Z}\right)$ as summarized by the Particle Data Group (PDG) 12], we obtain $\alpha_{s}\left(m_{Z}\right)_{\overline{\mathrm{MS}}}=0.116 \pm 0.0024$ (statistical error only) by excluding the data from the $Z$ parameter that will be discussed separately in this letter. The PDG assign an error of \pm 0.005 to account for the theoretical uncertainties. 
fits. One includes the leptonic asymmetries only and the other includes all the asymmetry measurements. For the combined fits $\chi_{\min }^{2}$ per degree of freedom is given. The deviation of each individual fit from the latter combined fit is given in the column $\chi$, defined as $\chi \equiv \frac{\left\langle\bar{s}^{2}\left(m_{Z}^{2}\right)\right\rangle-0.2306}{\Delta \bar{s}^{2}\left(m_{Z}^{2}\right)}$, where $\left\langle\bar{s}^{2}\left(m_{Z}^{2}\right)\right\rangle$ and $\Delta \bar{s}^{2}\left(m_{Z}^{2}\right)$ denote, respectively, the mean value and the error of $\bar{s}^{2}\left(m_{Z}^{2}\right)$ as determined from each data. The total $\chi^{2} /($ d.o.f.) is $9.4 / 5$ corresponding to $9 \%$ confidence level; this reflects the fact that the left-right asymmetry data from SLC gives a somewhat smaller value of $\bar{s}^{2}\left(m_{Z}^{2}\right)$ than the other data.

The only quantity which is sensitive to $\bar{g}_{Z}^{2}\left(m_{Z}^{2}\right)$ is the total width of the $Z$ boson, $\Gamma_{Z}$, which also depends on $\bar{s}^{2}\left(m_{Z}^{2}\right), \bar{\delta}_{b}$ and $\alpha_{s}$. On the other hand, $R_{\ell}$ and $\sigma_{h}^{0}$ depend on $\bar{s}^{2}\left(m_{Z}^{2}\right)$ and $\bar{\delta}_{b}$, and they depend strongly on $\alpha_{s}$. The ratios $R_{b}$ and $R_{c}$ are sensitive only to $\bar{\delta}_{b}$, that is, they hardly contribute to the universal parameter fits, but have little dependence upon $\alpha_{s}$. By taking $\bar{g}_{Z}^{2}\left(m_{Z}^{2}\right)$ and $\bar{s}^{2}\left(m_{Z}^{2}\right)$ as fit variables while treating $\bar{\delta}_{b}$ and $\alpha_{s}$ as external variables, we obtain from the two-parameter fit to all the $Z$ parameters :

$$
\begin{aligned}
& \left.\begin{array}{l}
\bar{g}_{Z}^{2}\left(m_{Z}^{2}\right)=0.55673-0.00056 \frac{\alpha_{s}+1.6 \bar{\delta}_{b}-0.100}{0.005} \pm 0.00087 \\
\bar{s}^{2}\left(m_{Z}^{2}\right)=0.23051+0.00008 \frac{\alpha_{s}+1.6 \delta_{b}-0.100}{0.005} \pm 0.00042
\end{array}\right\} \quad \rho_{\text {corr }}=0.28, \\
& \chi_{\min }^{2}=11.4+\left(\frac{\alpha_{s}+1.60 \bar{\delta}_{b}-0.1089}{0.0056}\right)^{2}+\left(\frac{\bar{\delta}_{b}+0.0015}{0.0046}\right)^{2},
\end{aligned}
$$

where the errors and the correlation are almost independent of $\alpha_{s}$ and $\bar{\delta}_{b}$. It should be noted that the quantities which are sensitive to $\alpha_{s}\left(\Gamma_{Z}, \sigma_{h}^{0}\right.$ and $\left.R_{\ell}\right)$ are also sensitive to $\bar{\delta}_{b}$, but they depend on the two parameters only through the combination

$$
\alpha_{s}^{\prime} \equiv \alpha_{s}\left(m_{Z}\right)+1.6 \bar{\delta}_{b}\left(m_{Z}^{2}\right) .
$$

This is because the hadronic contributions to these quantities arise from just one quantity, $\Gamma_{h}$, which depends on $\alpha_{s}$ and $\bar{\delta}_{b}$ in in approximately the above combination [7].

The results of the fit (2) are displayed in Fig. 1. We present 1- $\sigma$ allowed contours in the $\left(\bar{s}^{2}\left(m_{Z}^{2}\right), \bar{g}_{Z}^{2}\left(m_{Z}^{2}\right)\right)$ plane for $\alpha_{s}=0.116$ (solid lines) and 0.124 (dashed lines), and for $\bar{\delta}_{b}=-0.0100$ (thick lines) and -0.0079 (thin lines). Also shown by the lattices are the SM predictions for $125 \mathrm{GeV}<m_{t}<225 \mathrm{GeV}$ and $50 \mathrm{GeV}<m_{H}<1000 \mathrm{GeV}$. In these SM predictions all known two-loop corrections of the $\mathcal{O}\left(m_{t}^{4}\right)$ and $\mathcal{O}\left(\alpha \alpha_{s}\right)$ level have been included (see ref. [7] for references). We set $\alpha_{s}=0.116$ and $\delta_{\alpha}=0$ when calculating the SM prediction. In the SM the present estimate [13] of the hadronic contribution to the running of the charge form-factor, $\bar{\alpha}\left(q^{2}\right)$, is $\delta_{\alpha}=0 \pm 0.10$ [7]. While changing $\alpha_{s}$ by \pm 0.005 [12] has little effect, changing $\delta_{\alpha}$ by \pm 0.10 leads to a shift in the SM prediction for $\bar{s}^{2}\left(m_{Z}^{2}\right)$ by $\mp 0.00026$; this is more than half of its uncertainty.

So far we have treated $\bar{\delta}_{b}$ and $\alpha_{s}$ as external parameters. However, in principle, they can be extracted from the data once the remaining parameters are determined by other measurements, or they may be calculated in a specific theoretical model. 
The parameter $\bar{\delta}_{b}$ is determined from the ratio $R_{b}$ almost independently of the other parameters $\left(\bar{g}_{Z}^{2}\left(m_{Z}^{2}\right), \bar{s}^{2}\left(m_{Z}^{2}\right)\right.$ and $\left.\alpha_{s}\right)$, while its extraction from $\Gamma_{Z}, \sigma_{h}^{0}$ and $R_{\ell}$ depends more heavily on these parameters. We may parametrize $\bar{\delta}_{b}$ obtained from each measurement in terms of $\bar{g}_{Z}^{2}\left(m_{Z}^{2}\right), \bar{s}^{2}\left(m_{Z}^{2}\right)$ and $\alpha_{s}$ as follows :

$$
\bar{\delta}_{b}=\left\langle\bar{\delta}_{b}\right\rangle+C\left(\bar{g}_{Z}^{2}\right) \frac{\bar{g}_{Z}^{2}\left(m_{Z}^{2}\right)-0.55550}{0.00101}+C\left(\bar{s}^{2}\right) \frac{\bar{s}^{2}\left(m_{Z}^{2}\right)-0.23068}{0.00042}+C\left(\alpha_{s}\right) \frac{\alpha_{s}-0.116}{0.005} \pm \Delta \bar{\delta}_{b},
$$

where $\left\langle\bar{\delta}_{b}\right\rangle$ and $\Delta \bar{\delta}_{b}$ denote the mean value and the error of the individual measurement, respectively, while the coefficients $C\left(\bar{g}_{Z}^{2}\right), C\left(\bar{s}^{2}\right)$ and $C\left(\alpha_{s}\right)$ show its dependence on the remaining parameters. In Table 3 we show the results when $\bar{\delta}_{b}$ is extracted from $\Gamma_{Z}$, $\sigma_{h}^{0}, R_{\ell}$ and $R_{b}$. The same coefficient, $C\left(\alpha_{s}\right) \approx-0.0031$, appears for $\Gamma_{Z}, \sigma_{h}^{0}, R_{\ell}$, as a consequence of their dependence on the combination $\alpha_{s}^{\prime}$ (传). The present data on $R_{b}$ gives a value of $\bar{\delta}_{b}\left(m_{Z}^{2}\right)$ which is about 2 standard deviations larger than the SM prediction for $m_{t} \sim 175 \mathrm{GeV}\left(\bar{\delta}_{b} \sim-0.0100\right)$. It is further noted that, for $\alpha_{s}=0.116$, the data on $\Gamma_{Z}$

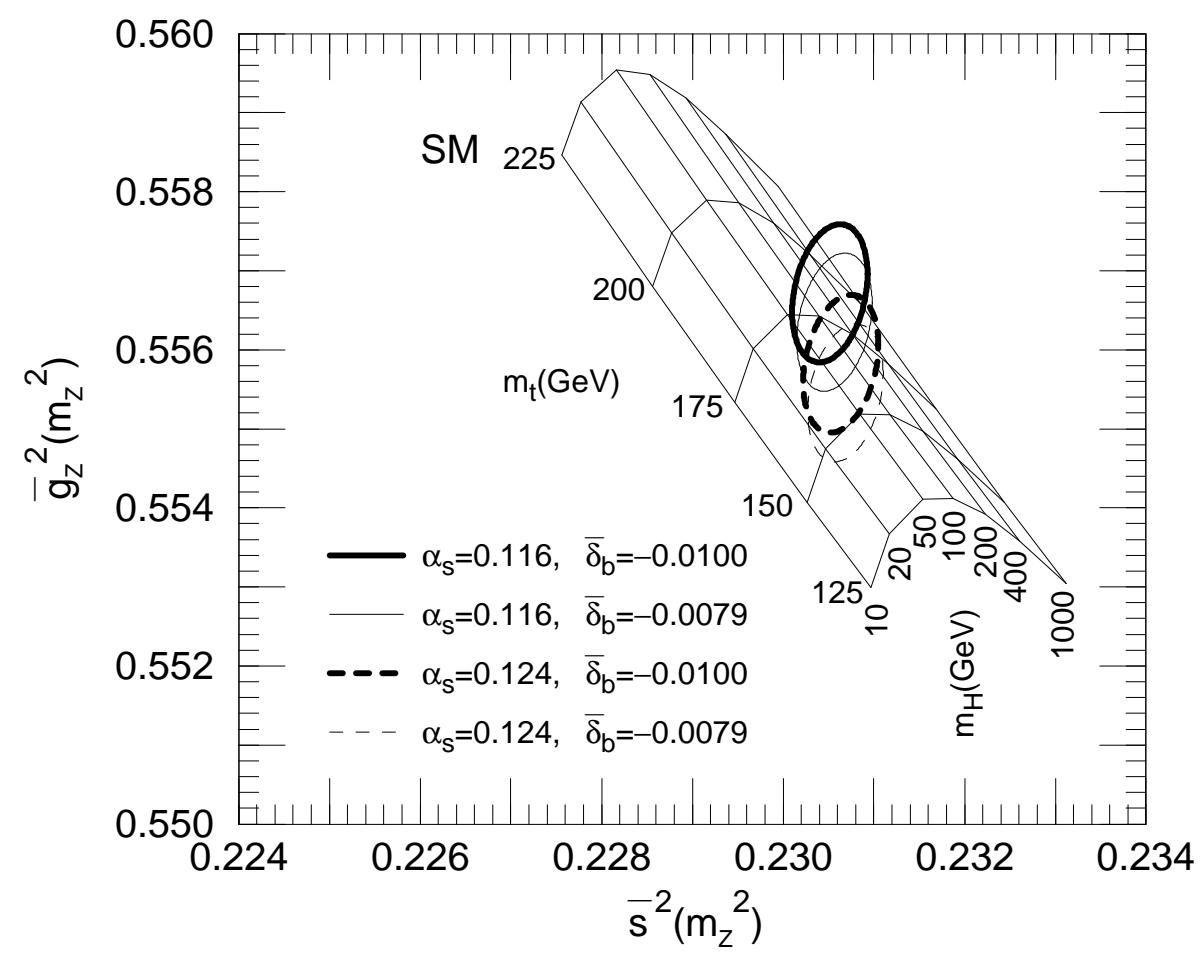

Fig. 1 A two-parameter fit to the $Z$ boson parameters in the $\left(\bar{s}^{2}\left(m_{Z}^{2}\right), \bar{g}_{Z}^{2}\left(m_{Z}^{2}\right)\right)$ plane. The $Z b_{L} b_{L}$ vertex form-factor, $\bar{\delta}_{b}\left(m_{Z}^{2}\right)$, and the QCD coupling, $\alpha_{s}\left(m_{Z}\right)$, are treated as external parameters. The 1- $\sigma$ contours are shown for two values of $\alpha_{s}\left(m_{Z}\right), 0.116$ (solid lines) and 0.124 (dashed lines), and for two values of $\bar{\delta}_{b}\left(m_{Z}^{2}\right),-0.0100$ (thick lines) and -0.0079 (thin lines). In the SM, $\bar{\delta}_{b}=-0.0100$ corresponds to $m_{t}=175 \mathrm{GeV}$, while $\bar{\delta}_{b}=-0.0079$ corresponds to $m_{t}=150 \mathrm{GeV}$. Also shown are the $\mathrm{SM}$ predictions in the range $125 \mathrm{GeV}<m_{t}<225 \mathrm{GeV}$ and $10 \mathrm{GeV}<m_{H}<1000 \mathrm{GeV}$, which are obtained by assuming $\alpha_{s}\left(m_{Z}\right)=0.116$ and $\delta_{\alpha} \equiv$ $1 / \bar{\alpha}\left(m_{Z}^{2}\right)-128.72=0$. 
Table $3 \quad \bar{\delta}_{b}\left(m_{Z}^{2}\right)$ as determined from various measurements. The dependences on the other parameters are shown by the coefficients $C\left(\bar{g}_{Z}^{2}\right), C\left(\bar{s}^{2}\right)$ and $C\left(\alpha_{s}\right)$. See eq. (四).

\begin{tabular}{|c|c|c|c|c|}
\hline measurements & $\left\langle\delta_{b}\right\rangle \pm \Delta \delta_{b}$ & $C\left(\bar{g}_{Z}^{2}\right)$ & $C\left(\bar{s}^{2}\right)$ & $C\left(\alpha_{s}\right)$ \\
\hline$\Gamma_{Z}$ & $-0.0036 \pm 0.0041$ & -0.00499 & +0.00118 & -0.00311 \\
$\sigma_{h}^{0}$ & $-0.0106 \pm 0.0142$ & - & +0.00021 & -0.00312 \\
$R_{\ell}$ & $-0.0036 \pm 0.0037$ & - & +0.00065 & -0.00311 \\
$R_{b}$ & $+0.0011 \pm 0.0051$ & - & -0.00004 & -0.00005 \\
\hline All & $-0.0034 \pm 0.0023$ & -0.00145 & +0.00065 & -0.00230 \\
\hline
\end{tabular}

Table $4 \alpha_{s} \equiv \alpha_{s}\left(m_{Z}\right) \overline{\mathrm{MS}}$ determined from the electroweak data by assuming the SM. The SM predictions for $\bar{g}_{Z}^{2}\left(m_{Z}^{2}\right), \bar{s}^{2}\left(m_{Z}^{2}\right)$ and $\bar{\delta}_{b}\left(m_{Z}^{2}\right)$ are also shown.

\begin{tabular}{ccccccc}
\hline$m_{t}(\mathrm{GeV})$ & $m_{H}(\mathrm{GeV})$ & $\bar{g}_{Z}^{2}\left(m_{Z}^{2}\right)$ & $\bar{s}^{2}\left(m_{Z}^{2}\right)$ & $\bar{\delta}_{b}\left(m_{Z}^{2}\right)$ & extracted $\alpha_{s}$ & $\chi_{\min }^{2} /($ d.o.f $)$ \\
\hline 150 & 100 & 0.55519 & 0.23117 & -0.0079 & $0.1253 \pm 0.0044$ & $15.2 / 10$ \\
150 & 1000 & 0.55408 & 0.23243 & -0.0079 & $0.1335 \pm 0.0044$ & $34.7 / 10$ \\
175 & 100 & 0.55644 & 0.23038 & -0.0100 & $0.1218 \pm 0.0044$ & $15.8 / 10$ \\
175 & 1000 & 0.55527 & 0.23167 & -0.0100 & $0.1303 \pm 0.0044$ & $21.1 / 10$ \\
\hline
\end{tabular}

and $R_{\ell}$ also give somewhat larger values of $\bar{\delta}_{b}$ than the SM prediction. Therefore we have to assume larger value of $\alpha_{s}\left(\alpha_{s} \sim 0.125\right)$ in order to get values of $\bar{\delta}_{b}$ consistent with the SM (See Table 4 ). Refering back to Table $\beta$, we give in the bottom line $\bar{\delta}_{b}$ as determined from all measurements of $Z$ parameters, that is, including asymmetries and $R_{c}$ together with the error correlations. It is clear that $\bar{\delta}_{b}$ is primarily determined from $\Gamma_{Z}$ and $R_{\ell}$, and hence it depends strongly on $\alpha_{s}$. An accurate measurement of $R_{b}$ offers the key to separate the measurements of $\alpha_{s}$ and $\bar{\delta}_{b}$.

Similarly, $\alpha_{s}$ can be extracted from the electroweak data alone once the form-factors $\bar{g}_{Z}^{2}\left(m_{Z}^{2}\right), \bar{s}^{2}\left(m_{Z}^{2}\right)$ and $\bar{\delta}_{b}\left(m_{Z}^{2}\right)$ are given by a specific model. From the global fit we find

$\alpha_{s}=0.1150-0.0032 \frac{\bar{g}_{Z}^{2}\left(m_{Z}^{2}\right)-0.55550}{0.00101}+0.0015 \frac{\bar{s}^{2}\left(m_{Z}^{2}\right)-0.23068}{0.00042}-0.0042 \frac{\bar{\delta}_{b}+0.0034}{0.0026} \pm 0.0044$. (5)

Here the reference values, $\bar{g}_{Z}^{2}\left(m_{Z}^{2}\right)=0.55550, \bar{s}^{2}\left(m_{Z}^{2}\right)=0.23068$ and $\bar{\delta}_{b} \equiv \bar{\delta}_{b}\left(m_{Z}^{2}\right)=-0.0034$, are the best-fit values when we make a three-parameter fit in terms of $\left(\bar{g}_{Z}^{2}\left(m_{Z}^{2}\right), \bar{s}^{2}\left(m_{Z}^{2}\right), \bar{\delta}_{b}\right)$. The quantities that are most sensitive to $\alpha_{s}$ are $\Gamma_{Z}$ and $R_{\ell}$. In fact, one can obtain the above results (5) by solving eq. (4) for $\Gamma_{Z}$ and $R_{\ell}$ using the values in Table 3 . In the SM the form-factors $\bar{g}_{Z}^{2}\left(m_{Z}^{2}\right), \bar{s}^{2}\left(m_{Z}^{2}\right)$ and $\bar{\delta}_{b}\left(m_{Z}^{2}\right)$ are functions of $m_{t}$ and $m_{H}$, and hence $\alpha_{s}$ can be determined as a function of $m_{t}$ and $m_{H}$. Table 4 shows the extracted values of $\alpha_{s}$ for several set of $m_{t}$ and $m_{H}$ with $\delta_{\alpha}=0$, together with the SM prediction for the three form-factors. For the given sets of $m_{t}$ and $m_{H}$ in the table the extracted value of $\alpha_{s}$ is somewhat larger than the estimate of the PDG [12 * which is $\alpha_{s}=0.116 \pm 0.005$. 
If we allow all the four parameters, $\bar{g}_{Z}^{2}\left(m_{Z}^{2}\right), \bar{s}^{2}\left(m_{Z}^{2}\right), \bar{\delta}_{b}\left(m_{Z}^{2}\right)$ and $\alpha_{s}$, to be fitted by the data we find

$$
\left.\begin{array}{rl}
\bar{g}_{Z}^{2}\left(m_{Z}^{2}\right) & =0.55570 \pm 0.00108 \\
\bar{s}^{2}\left(m_{Z}^{2}\right) & =0.23066 \pm 0.00043 \\
\bar{\delta}_{b}\left(m_{Z}^{2}\right) & =-0.0017 \pm 0.0049 \\
\alpha_{s} & =0.1117 \pm 0.0093
\end{array}\right\} \quad \rho_{\text {corr }}=\left(\begin{array}{rrrr}
1.00 & 0.10 & 0.01 & -0.36 \\
& 1.00 & 0.01 & 0.12 \\
& & 1.00 & -0.80 \\
& & & 1.00
\end{array}\right)
$$

This is, of course, consistent with the parametrization (2). The rather small mean value for $\alpha_{s}$ is a consequence of a large $\bar{\delta}_{b}$ which is prefered by the data on $R_{b}$, as explained above (see eq.(2b) and Table [3).

It is often important to obtain the constraint on the number of neutrinos, $N_{\nu}$, which, in the above analysis, has been assumed to be $N_{\nu}=3$. We consider here an analysis without the condition, $N_{\nu}=3$. By treating $N_{\nu}, \bar{g}_{Z}^{2}\left(m_{Z}^{2}\right)$ and $\bar{s}^{2}\left(m_{Z}^{2}\right)$ as the three parameters of our fit we obtain :

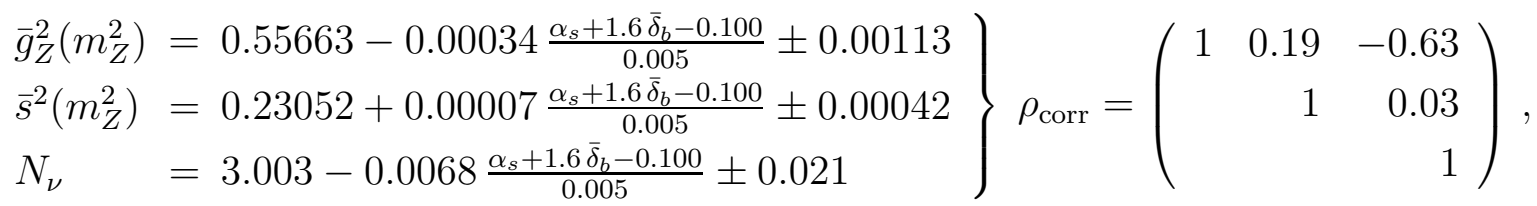

$$
\begin{aligned}
& \chi_{\min }^{2}=11.2+\left(\frac{\alpha_{s}+1.60 \bar{\delta}_{b}-0.1099}{0.0060}\right)^{2}+\left(\frac{\bar{\delta}_{b}+0.0015}{0.0046}\right)^{2} .
\end{aligned}
$$

This result strongly supports the validity of the assumption (a) in page 6 . We can also find the best-fit value of $N_{\nu}$ from the above result as functions of $\bar{g}_{Z}^{2}\left(m_{Z}^{2}\right), \bar{s}^{2}\left(m_{Z}^{2}\right), \bar{\delta}_{b}\left(m_{Z}^{2}\right)$ and $\alpha_{s}$ :

$$
\begin{aligned}
N_{\nu}= & 2.993-0.0128 \frac{\bar{g}_{Z}^{2}\left(m_{Z}^{2}\right)-0.55550}{0.00101}+0.0035 \frac{\bar{s}^{2}\left(m_{Z}^{2}\right)-0.23068}{0.00042} \\
& -0.0098 \frac{\bar{\delta}_{b}\left(m_{Z}^{2}\right)+0.0034}{0.0026}-0.0118 \frac{\alpha_{s}-0.116}{0.005} \pm 0.016,
\end{aligned}
$$

where the reference values for the three form-factors are chosen as in eq. (5).

The above results may be re-interpreted in the language of $S, T$ and $U$ [7, 8]. When the new physics scale is higher than the scale of precision measurements new-physics contributions to the running of the charge form-factors may be neglected. In such a case one may combine the low-energy neutral-current experiments which determine the formfactors $\bar{g}_{Z}^{2}(0)$ and $\bar{s}^{2}(0)[7]$ with the $Z$ parameter measurements. Here one assumes that the running of $\bar{g}_{Z}^{2}\left(q^{2}\right)$ and $\bar{s}^{2}\left(q^{2}\right)$ between $q^{2}=0$ and $q^{2}=m_{Z}^{2}$ is governed only by SM physics. The universal propagator corrections in the neutral-current sector are then parametrized by just two parameters, essentially $S$ and $T$. The $U$ parameter is determined from the 
charged-current sector through the charge form-factor $\bar{g}_{W}^{2}(0)$ [7] which is determined from measurements of the $W$-boson mass using the relation

$$
G_{F}=\frac{\bar{g}_{W}^{2}(0)+\hat{g}^{2} \bar{\delta}_{G}}{4 \sqrt{2} m_{W}^{2}} .
$$

Here $\bar{\delta}_{G}$ is the vertex and box correction to the muon lifetime 14 after subtraction of the pinch term. In the SM, $\bar{\delta}_{G}=0.0055$ [7]. We adopt a modified version of the original $S, T$ and $U$ parameters which includes the SM radiative effects as well as new physics contributions [7, 11]. They are related to the charge form-factors by the following identities [7]:

$$
\begin{aligned}
\frac{1}{\bar{g}_{Z}^{2}(0)} & =\frac{1+\bar{\delta}_{G}-\alpha T}{4 \sqrt{2} G_{F} m_{Z}^{2}}, \\
\bar{s}^{2}\left(m_{Z}^{2}\right) & =\frac{1}{2}-\sqrt{\frac{1}{4}-\bar{\alpha}^{2}\left(m_{Z}^{2}\right)\left(\frac{4 \pi}{\bar{g}_{Z}^{2}(0)}+\frac{S}{4}\right)}, \\
\frac{4 \pi}{\bar{g}_{W}^{2}(0)} & =\frac{\bar{s}^{2}\left(m_{Z}^{2}\right)}{\bar{\alpha}^{2}\left(m_{Z}^{2}\right)}-\frac{1}{4}(S+U) .
\end{aligned}
$$

It is clear from eqs. (10) that $\bar{g}_{Z}^{2}(0)$ is dependent upon $\bar{\delta}_{G}-\alpha T, \bar{s}^{2}\left(m_{Z}^{2}\right)$ is dependent upon $\bar{g}_{Z}^{2}(0), \bar{\alpha}\left(m_{Z}^{2}\right)$ and $S$, and $\bar{g}_{W}^{2}(0)$ is dependent upon $\bar{s}^{2}\left(m_{Z}^{2}\right), \bar{\alpha}\left(m_{Z}^{2}\right)$ and $S+U$. It is instructive to express these form-factors as approximate linear combinations of $S, T$ and $U$. By inserting $1 / \bar{\alpha}\left(m_{Z}^{2}\right) \equiv 4 \pi / \bar{e}\left(m_{Z}^{2}\right)=128.72+\delta_{\alpha}$, we find

$$
\begin{aligned}
\bar{g}_{Z}^{2}(0) & =0.5456 \quad+0.0040\left(T+\frac{0.0055-\bar{\delta}_{G}}{\alpha}\right), \\
\bar{s}^{2}\left(m_{Z}^{2}\right) & =0.2334+0.0036 S-0.0024\left(T+\frac{0.0055-\bar{\delta}_{G}}{\alpha}\right) \\
\bar{g}_{W}^{2}(0) & =0.4183-0.0030 S+0.0044\left(T+\frac{0.0055-\bar{\delta}_{G}}{\alpha}\right)+0.0035 U+0.0014 \delta_{\alpha} .
\end{aligned}
$$

Here we explicitly retain $\delta_{\alpha}$ and $\bar{\delta}_{G}$ in the expansion. The values of the charge formfactors $\bar{g}_{Z}^{2}\left(m_{Z}^{2}\right)$ and $\bar{s}^{2}(0)$ are then calculated from $\bar{g}_{Z}^{2}(0)$ and $\bar{s}^{2}\left(m_{Z}^{2}\right)$ above, respectively, by assuming the SM running [7] of the form factors between $q^{2}=0$ and $q^{2}=m_{Z}^{2}$.

In Table 5, we give a list of the data from the low-energy neutral-current experiments that we use in our analysis. They are neutrino-nucleon scattering $\left(\nu_{\mu}-q\right)$, neutrino-electron scattering $\left(\nu_{\mu}-e\right)$, atomic parity violation (APV) and polarized electron-deuteron scattering $(e-\mathrm{D})$ experiments. Additionally, the $W$ mass data [5] is given. See ref. [7] for details. Also shown are the SM predictions for $\left(m_{t}, m_{H}\right)=(150,100),(150,1000),(175,100)$ and $(175,1000)$ in $\mathrm{GeV}$ units for $\alpha_{s}=0.116$ and $\delta_{\alpha}=0$.

From the low-energy neutral-current experiments we obtain two universal parameters, $\bar{g}_{Z}^{2}(0)$ and $\bar{s}^{2}(0)$, which may be reparametrized in terms of $\bar{g}_{Z}^{2}\left(m_{Z}^{2}\right)$ and $\bar{s}^{2}\left(m_{Z}^{2}\right)$ by assuming 
Table 5 Data from low-energy neutral-current experiments and $W$-mass measurements that are used in our analysis. The SM prediction is also shown.

\begin{tabular}{|c|c|c|c|c|c|}
\hline measurement & data & SM pre & iction (c & $=0.116$ & $\left.\overline{\delta_{\alpha}}=0\right)$ \\
\hline$m_{t}(\mathrm{GeV})$ & & 150 & 150 & 175 & 175 \\
\hline$m_{H}(\mathrm{GeV})$ & & 100 & 1000 & 100 & 1000 \\
\hline$\nu-q[\overline{0}, 15]$ & & & & & \\
\hline$g_{L}^{2}$ & $0.2980 \pm 0.0044$ & 0.2976 & 0.2955 & 0.2995 & 0.2973 \\
\hline$g_{R}^{2}$ & $0.0307 \pm 0.0047$ & 0.0296 & 0.0298 & 0.0295 & 0.0297 \\
\hline & $-0.0589 \pm 0.0237$ & -0.0633 & -0.0632 & -0.0634 & -0.0634 \\
\hline & $0.0206 \pm 0.0160$ & 0.0177 & 0.0178 & 0.0177 & 0.0178 \\
\hline$\chi^{2} /($ d.o.f. $)$ & & $0.21 / 4$ & $0.77 / 4$ & $0.25 / 4$ & $0.25 / 4$ \\
\hline$\nu-e$ 16 & & & & & \\
\hline$s_{e f f}^{2}$ & $0.233 \pm 0.008$ & 0.231 & 0.232 & 0.230 & 0.231 \\
\hline$\rho_{\text {eff }}$ & $1.007 \pm 0.028$ & 1.011 & 1.009 & 1.013 & 1.011 \\
\hline$\chi^{2} /($ d.o.f. $)$ & & $0.09 / 2$ & $0.02 / 2$ & $0.18 / 2$ & $0.06 / 2$ \\
\hline APV 17 & & & & & \\
\hline$Q_{W}$ & $-71.04 \pm 1.81$ & -73.20 & -73.30 & -73.20 & -73.30 \\
\hline$\chi^{2} /($ d.o.f. $)$ & & $1.42 / 1$ & $1.56 / 1$ & $1.43 / 1$ & $1.57 / 1$ \\
\hline$e^{-\mathrm{D}}$,7, 18, & & & & & \\
\hline $2 C_{1 u}-C_{1 d}$ & $0.938 \pm 0.264$ & 0.719 & 0.713 & 0.723 & 0.717 \\
\hline $2 C_{2 u}-C_{2 d}$ & $-0.659 \pm 1.228$ & 0.099 & 0.092 & 0.104 & 0.096 \\
\hline$\chi^{2} /($ d.o.f. $)$ & & $1.43 / 2$ & $1.69 / 2$ & $1.27 / 2$ & $1.51 / 2$ \\
\hline$W$ mass [5, 12] & & & & & \\
\hline$m_{W}$ & $80.24 \pm 0.16$ & 80.25 & 80.08 & 80.40 & 80.23 \\
\hline$\chi^{2} /($ d.o.f. $)$ & & $0.00 / 1$ & $0.96 / 1$ & $1.04 / 1$ & $0.00 / 1$ \\
\hline
\end{tabular}

the SM running between $q^{2}=0$ and $q^{2}=m_{Z}^{2}$. In Table 6 we show the two universal parameters, $\bar{g}_{Z}^{2}\left(m_{Z}^{2}\right)$ and $\bar{s}^{2}\left(m_{Z}^{2}\right)$, determined from $\nu_{\mu}-f$ and $e-q$ sectors and from all four experiments. We used $m_{t}=175 \mathrm{GeV}$ and $m_{H}=100 \mathrm{GeV}$ when calculating the SM running, but the results are insensitive to these values in the region $m_{t}>100 \mathrm{GeV}$ and $m_{H}>100 \mathrm{GeV}$.

The universal electroweak parameter, $\bar{g}_{W}^{2}(0)$, is obtained from eq. (9) by combining the data on $m_{W}$ with the muon life-time parameter, $G_{F}$. We find

$$
\bar{g}_{W}^{2}(0)=0.4225-0.0031 \frac{\bar{\delta}_{G}-0.0055}{\alpha} \pm 0.0017 .
$$

Now we may combine all the electroweak data; eq. (2), Table 6 and eq. (12). Treating $\bar{\delta}_{b}, \alpha_{s}$ and $\delta_{\alpha}$ as external parameters, and by setting $\bar{\delta}_{G}=0.0055$, we find from the 
Table $6 \bar{g}_{Z}^{2}\left(m_{Z}^{2}\right)$ and $\bar{s}^{2}\left(m_{Z}^{2}\right)$ determined from low-energy neutral-current experiments. The running of the charge form-factor are calculated in the SM with $m_{t}=175 \mathrm{GeV}$ and $m_{H}=$ $100 \mathrm{GeV}$.

\begin{tabular}{|c|ccrc|}
\hline & $\bar{g}_{Z}^{2}\left(m_{Z}^{2}\right)$ & $\bar{s}^{2}\left(m_{Z}^{2}\right)$ & $\rho_{\text {corr }}$ & $\chi_{\min }^{2} /$ (d.o.f. $)$ \\
\hline$\nu_{\mu}-f\left(\nu_{\mu}-e+\nu_{\mu}-q\right)$ & $0.5568 \pm 0.0048$ & $0.2331 \pm 0.0072$ & 0.75 & $0.19 / 4$ \\
$e-q\left(\mathrm{APV}+e^{-\mathrm{D})}\right.$ & $0.5583 \pm 0.0170$ & $0.2188 \pm 0.0093$ & -0.62 & $0.46 / 1$ \\
\hline All $\left(\nu_{\mu}-f+e-q\right)$ & $0.5533 \pm 0.0037$ & $0.2266 \pm 0.0047$ & 0.53 & $2.22 / 7$ \\
\hline
\end{tabular}

three-parameter fit :

$$
\begin{aligned}
& \left.\begin{array}{rrr}
S= & -0.18-0.06 \frac{\alpha_{s}+1.6 \bar{\delta}_{b}-0.100}{0.005}+0.07 \frac{\delta_{\alpha}}{0.10} \pm 0.20 \\
T= & 0.93-0.13 \frac{\alpha_{s}+1.6 \bar{\delta}_{b}-0.100}{0.005} & \pm 0.20 \\
U= & -0.12+0.10 \frac{\alpha_{s}+1.6 \bar{\delta}_{b}-0.100}{0.005}+0.02 \frac{\delta_{\alpha}}{0.10} \pm 0.50
\end{array}\right\} \quad \rho_{\text {corr }}=\left(\begin{array}{rrr}
1 & 0.84 & -0.08 \\
& -0.22 \\
& 1
\end{array}\right) \\
& \chi_{\min }^{2}=14.4+\left(\frac{\alpha_{s}+1.60 \bar{\delta}_{b}-0.1091}{0.0055}\right)^{2}+\left(\frac{\bar{\delta}_{b}+0.0015}{0.0046}\right)^{2} .
\end{aligned}
$$

The dependence of the $S$ and $U$ parameters upon $\delta_{\alpha}$ may be understood from eq. (11). For an arbitrary value of $\bar{\delta}_{G}$ the fitted value of $T$ should be shifted by $\left(\bar{\delta}_{G}-0.0055\right) / \alpha$. It should be noted that the uncertainty in $S$ coming from $\delta_{\alpha}=0 \pm 0.1$ is of the same order as from the uncertainty in $\alpha_{s}$; they are not negligible when compared to the overall error. The $T$ parameter has little $\delta_{\alpha}$ dependence, but it is sensitive to $\alpha_{s}$.

The above results are shown in Fig. 2 by the $1-\sigma$ contours in the $(S, T)$ plane. Four cases are shown : $\alpha_{s}=0.116$ (solid lines) and 0.124 (dashed lines) with $\bar{\delta}_{b}=-0.0100$ (thick lines) and -0.0079 (thin lines). As for the running of the charge form-factors $\bar{s}^{2}\left(q^{2}\right)$ and $\bar{g}_{Z}^{2}\left(q^{2}\right)$ between $q^{2}=0$ and $q^{2}=m_{Z}^{2}$, we set $m_{H}=100 \mathrm{GeV}$, and use values of $m_{t}$ corresponding to the above values of $\bar{\delta}_{b}\left(m_{t}=175 \mathrm{GeV}\right.$ for $\bar{\delta}_{b}=-0.0100$ and $m_{t}=150 \mathrm{GeV}$ for $\left.\bar{\delta}_{b}=-0.0079\right)$. The SM predictions are also shown in Fig. 2 by lattices in the region $125 \mathrm{GeV}<m_{t}<225 \mathrm{GeV}$ and $50 \mathrm{GeV}<m_{H}<1000 \mathrm{GeV}$. The estimates [8] of $S$ and $T$ for the minimal (one-doublet) $\mathrm{SU}\left(N_{c}\right)$ Technicolor (TC) models with $N_{c}=2,3,4$ are also shown in the figure. It is clearly seen that the current experiments provide a fairly stringent constraint on the simple TC models if a QCD-like spectrum and the large $N_{c}$ scaling are assumed [8]. Only with a positive value for $\delta_{\alpha}$ and a small value of $\alpha_{s}^{\prime} \equiv \alpha_{s}+1.6 \bar{\delta}_{b}$ can the $N_{c}=2$ one-doublet TC model be made consistent with the data.

For definiteness we provide, in Table 7, the values of $S, T$ and $U$ after the SM contributions are subtracted $\left(S_{\text {new }} \equiv S-S_{\mathrm{SM}}\right.$, etc.). The dependence upon $m_{t}$ and $m_{H}$ of $S, T$ and $U$ appears since we have assumed the SM running of the charge form-factors which in 
turn depends slightly on $m_{t}$ and $m_{H}$; also because we have used the SM prediction for $\bar{\delta}_{b}$ which depends strongly on $m_{t}$. All values in the table are obtained by setting $\alpha_{s}=0.116$ and $\delta_{\alpha}=0$. The values for different values of $\alpha_{s}$ and $\delta_{\alpha}$ together with the error correlation matrix can be 'read-off' from eq. (13).

Finally we discuss the constraints on $m_{t}$ and $m_{H}$ from all the data in Tables 11 and 5 in the minimal SM. In this case all the form-factors, $\bar{g}_{Z}^{2}\left(m_{Z}^{2}\right), \bar{s}^{2}\left(m_{Z}^{2}\right), \bar{g}_{Z}^{2}(0), \bar{s}^{2}(0), \bar{g}_{W}^{2}(0)$ and $\bar{\delta}_{b}\left(m_{Z}^{2}\right)$, depend uniquely on the two mass parameters, $m_{t}$ and $m_{H}$. Consequently the results of the fits for these form-factors constraint $m_{t}$ and $m_{H}$.

Fig. 3 shows the result of the global fit to all electroweak data in the $\left(m_{H}, m_{t}\right)$ plane for $\alpha_{s}=0.116$ and 0.124 with $\delta_{\alpha}=0$. Here $\alpha_{s}=0.116$ is the mean value of the PDG listing [12] and $\alpha_{s}=0.124$ is the best SM fit value to all electroweak data in our three-

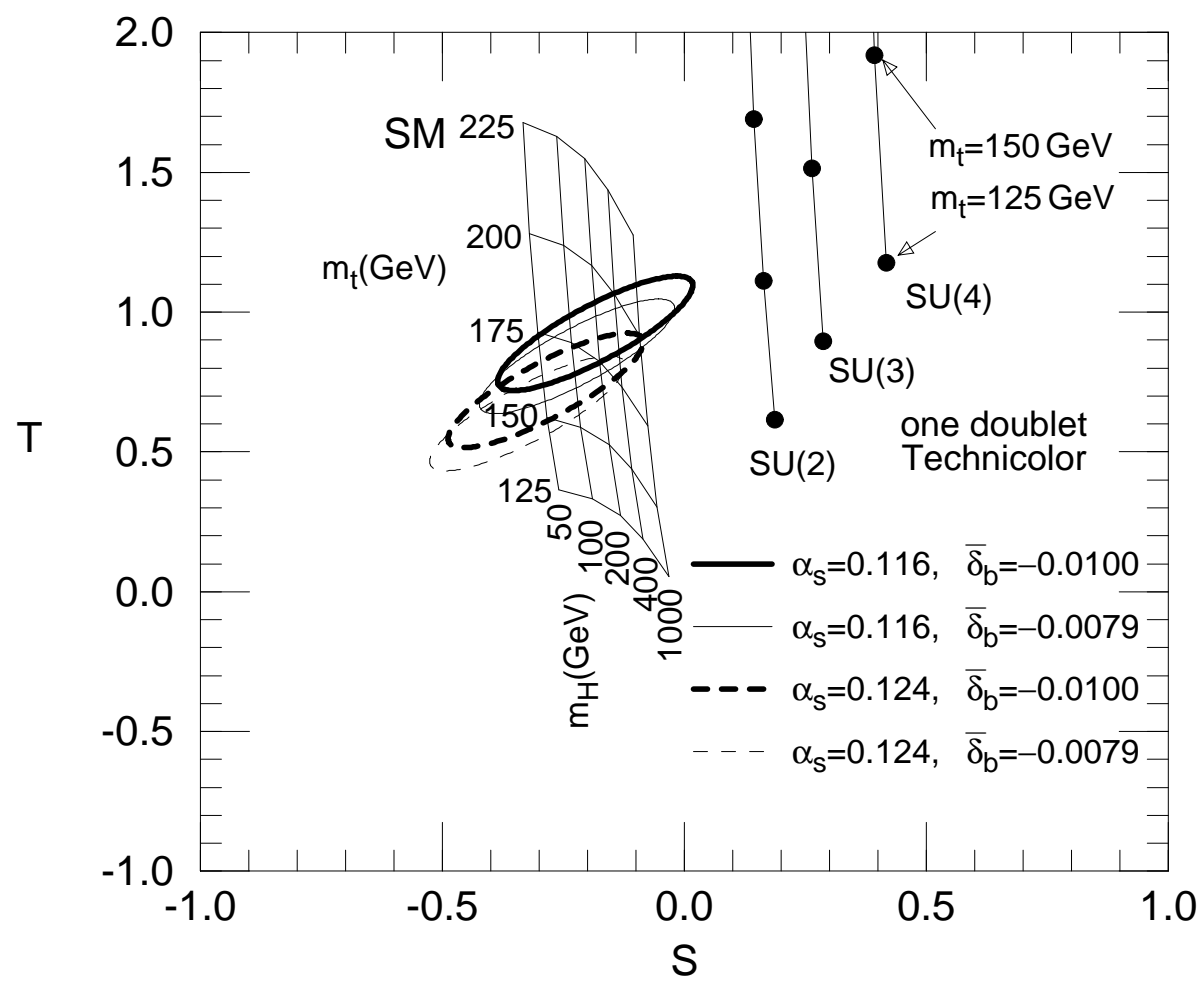

Fig. 2 A two-parameter fit to the $Z$ boson parameters in the $(S, T)$ plane for $\delta_{\alpha}=0$ and $\bar{\delta}_{G}=0.0055$. The $Z b_{L} b_{L}$ vertex form-factor, $\bar{\delta}_{b}\left(m_{Z}^{2}\right)$, and the QCD coupling, $\alpha_{s}\left(m_{Z}\right)$, are treated as external parameters in the fit. The 1- $\sigma$ contours are shown for two values of $\alpha_{s}, 0.116$ (solid lines) and 0.124 (dashed lines), and for two values of $\bar{\delta}_{b},-0.0100$ (thick lines) and -0.0079 (thin lines). In the SM, $\bar{\delta}_{b}=-0.0100$ corresponds to $m_{t}=175 \mathrm{GeV}$, while $\bar{\delta}_{b}=-0.0079$ corresponds to $m_{t}=150 \mathrm{GeV}$. Also shown are the $\mathrm{SM}$ predictions in the range $125 \mathrm{GeV}<m_{t}<225 \mathrm{GeV}$ and $50 \mathrm{GeV}<m_{H}<1000 \mathrm{GeV}$. The mild dependence on $m_{t}$ and $m_{H}$ in the SM running between $q^{2}=0$ and $q^{2}=m_{Z}^{2}$ is calculated with $m_{H}=100 \mathrm{GeV}$ and the above values of $m_{t}$ determined from $\bar{\delta}_{b}\left(m_{Z}^{2}\right)$. The estimates [8] for one doublet $\mathrm{SU}\left(N_{c}\right)$-TC models are shown for $N_{c}=2,3,4$. 
Table 7 Constraints on the parameters $S_{\text {new }}, T_{\text {new }}, U_{\text {new }}$ which are obtained by subtracting the SM contribution $S_{\mathrm{SM}}, T_{\mathrm{SM}}, U_{\mathrm{SM}}$ from $S, T, U$ for $\alpha_{s}=0.116$ and $\delta_{\alpha}=0$. Correlations among errors are the same as in eq. (13a).

\begin{tabular}{|c|c|c|c|c|c|}
\hline $\begin{array}{c}m_{t} \\
(\mathrm{GeV})\end{array}$ & $\begin{array}{c}m_{H} \\
(\mathrm{GeV})\end{array}$ & $\left(\begin{array}{l}S \\
T \\
U\end{array}\right)$ & $\left(\begin{array}{c}S_{\mathrm{SM}} \\
T_{\mathrm{SM}} \\
U_{\mathrm{SM}}\end{array}\right)$ & $\left(\begin{array}{l}S_{\text {new }} \\
T_{\text {new }} \\
U_{\text {new }}\end{array}\right)$ & $\chi_{\min }^{2} /($ d.o.f. $)$ \\
\hline \multirow{3}{*}{150} & \multirow{3}{*}{100} & $-0.22 \pm 0.20$ & -0.21 & $-0.01 \pm 0.20$ & \multirow{3}{*}{$17.5 / 18$} \\
\hline & & $0.84 \pm 0.20$ & 0.59 & $0.25 \pm 0.20$ & \\
\hline & & $-0.05 \pm 0.50$ & 0.30 & $-0.35 \pm 0.50$ & \\
\hline \multirow{3}{*}{150} & \multirow{3}{*}{1000} & $-0.23 \pm 0.20$ & -0.06 & $-0.17 \pm 0.20$ & \multirow{3}{*}{$17.6 / 18$} \\
\hline & & $0.83 \pm 0.20$ & 0.30 & $0.53 \pm 0.20$ & \\
\hline & & $-0.05 \pm 0.50$ & 0.29 & $-0.34 \pm 0.50$ & \\
\hline \multirow{3}{*}{175} & \multirow{3}{*}{100} & $-0.18 \pm 0.20$ & -0.23 & $0.05 \pm 0.20$ & \multirow{3}{*}{$20.6 / 18$} \\
\hline & & $0.92 \pm 0.20$ & 0.89 & $0.03 \pm 0.20$ & \\
\hline & & $-0.12 \pm 0.50$ & 0.36 & $-0.48 \pm 0.50$ & \\
\hline \multirow{3}{*}{175} & \multirow{3}{*}{1000} & $-0.19 \pm 0.20$ & -0.08 & $-0.12 \pm 0.20$ & \multirow{3}{*}{$20.7 / 18$} \\
\hline & & $0.91 \pm 0.20$ & 0.59 & $0.32 \pm 0.20$ & \\
\hline & & $-0.12 \pm 0.50$ & 0.35 & $-0.47 \pm 0.50$ & \\
\hline
\end{tabular}

parameter fit in terms of $m_{t}, m_{H}$ and $\alpha_{s}$. The thick inner and outer contours correspond to $\Delta \chi^{2} \equiv \chi^{2}-\chi_{\min }^{2}=1(\sim 39 \% \mathrm{CL})$, and $\Delta \chi^{2}=4.61(\sim 90 \% \mathrm{CL})$, respectively. The minimum of $\chi^{2}$ is indicated by an " $\times$ ". The corresponding value of $\chi_{\min }^{2}$ is 19.8 for $\alpha_{s}=0.116(\mathrm{a})$ and 16.8 for $\alpha_{s}=0.124$ (b). We also give the separate $1-\sigma$ constraints arising from the $Z$-pole asymmetries, $\Gamma_{Z}$, and $m_{W}$. The asymmetries constrain $m_{t}$ and $m_{H}$ through $\bar{s}^{2}\left(m_{Z}^{2}\right)$ while $\Gamma_{Z}$ constrains them through the three form-factors $\bar{g}_{Z}^{2}\left(m_{Z}^{2}\right)$, $\bar{s}^{2}\left(m_{Z}^{2}\right)$ and $\bar{\delta}_{b}\left(m_{Z}^{2}\right)$. In other words, the asymmetries measure the combination of $S$ and $T$ as in eq. (11b); both of $S$ and $T$ are functions of $m_{t}$ and $m_{H}$. On the other hand, $\Gamma_{Z}$ measures a different combination of $S$ and $T$ with an additional constraint from $\bar{\delta}_{b}$. A remarkable point apparent from Fig. 3 is that, in the SM, when $m_{t}$ and $m_{H}$ are much larger than $m_{Z}, \Gamma_{Z}$ depends upon almost the same combination of $m_{t}$ and $m_{H}$ as the one measured through $\bar{s}^{2}\left(m_{Z}^{2}\right)$. This is because $\bar{g}_{Z}^{2}\left(m_{Z}^{2}\right)$ has a quadratic dependence on $m_{t}$ which is positive while the quadratic dependence on $m_{t}$ of $\bar{\delta}_{b}$ is negative, and these two effects largely cancel. The result is that the ratio of dependences of $\Gamma_{Z}$ on $m_{t}$ and $m_{H}$ are similar to the case of of $\bar{s}^{2}\left(m_{Z}^{2}\right)$. Because of this only a band of $m_{t}$ and $m_{H}$ can be strongly constrained from the asymmetries and $\Gamma_{Z}$ alone, despite their very small experimental errors. The constraint from the data on $m_{W}$ overlaps this allowed region.

Quantities which help to disentangle the above $m_{t}-m_{H}$ correlation are $R_{\ell}$ and $R_{b}$. The constraints from these two data are shown in Fig. 3 by dashed lines corresponding to 
$\sqrt{\chi^{2}}=1.5,2.0,2.5,3.0$. As shown in Fig. 4 the SM prediction for $R_{\ell}$ is very sensitive to the assumed value of $\alpha_{s}$, and, for $\alpha_{s}=0.116$, the data favors the region where both $m_{t}$ and $m_{H}$ are small; for $\alpha_{s}=0.124$ it does not provide a stringent limit on $m_{t}$ and $m_{H}$. This explains the difference between the two cases, $\alpha_{s}=0.116$ (Fig. Ba) and 0.124 (Fig. 3 $\mathrm{b}$ ). On the other hand, data on $R_{b}$ favors small $m_{t}$ almost independently of $m_{H}$. It is hence the $R_{\ell}$ and $R_{b}$ data that constrain the values of $m_{t}$ and $m_{H}$ from above. If not for the data on $R_{\ell}$ and $R_{b}$, the common shaded region in Fig. 3 with very large $m_{H}$ $\left(m_{H} \sim 1 \mathrm{TeV}\right)$ could not be excluded by the electroweak data alone.

The results of the fits for different values of $\delta_{\alpha}$ are shown in Fig 5 . The case for $\delta_{\alpha}=-0.1$ is shown by dashed lines, and the case for $\delta_{\alpha}=+0.1$ is shown by solid lines. In both cases the inner and outer contours correspond to $\Delta \chi^{2}=1(\sim 39 \% \mathrm{CL})$, and $\Delta \chi^{2}=4.61(\sim 90 \% \mathrm{CL})$, respectively.

If the lower bound for $m_{H}\left(m_{H}>63 \mathrm{GeV}\right.$ at $\left.95 \% \mathrm{CL}\right)$ measured by the LEP experiments [19] is imposed then $m_{t}$ below $130 \mathrm{GeV}$ is clearly disfavored for these regions on $\alpha_{s}$ and $\delta_{\alpha}$. This agrees with the directly established lower top-mass limit [20,21].

The $\chi^{2}$ function of the global fit to all electroweak data can be parametrized in terms of the four parameters $m_{t}, m_{H}, \alpha_{s}$ and $\delta_{\alpha}$ together with the constraint $\delta_{\alpha}=0.0 \pm 0.1$ [13]
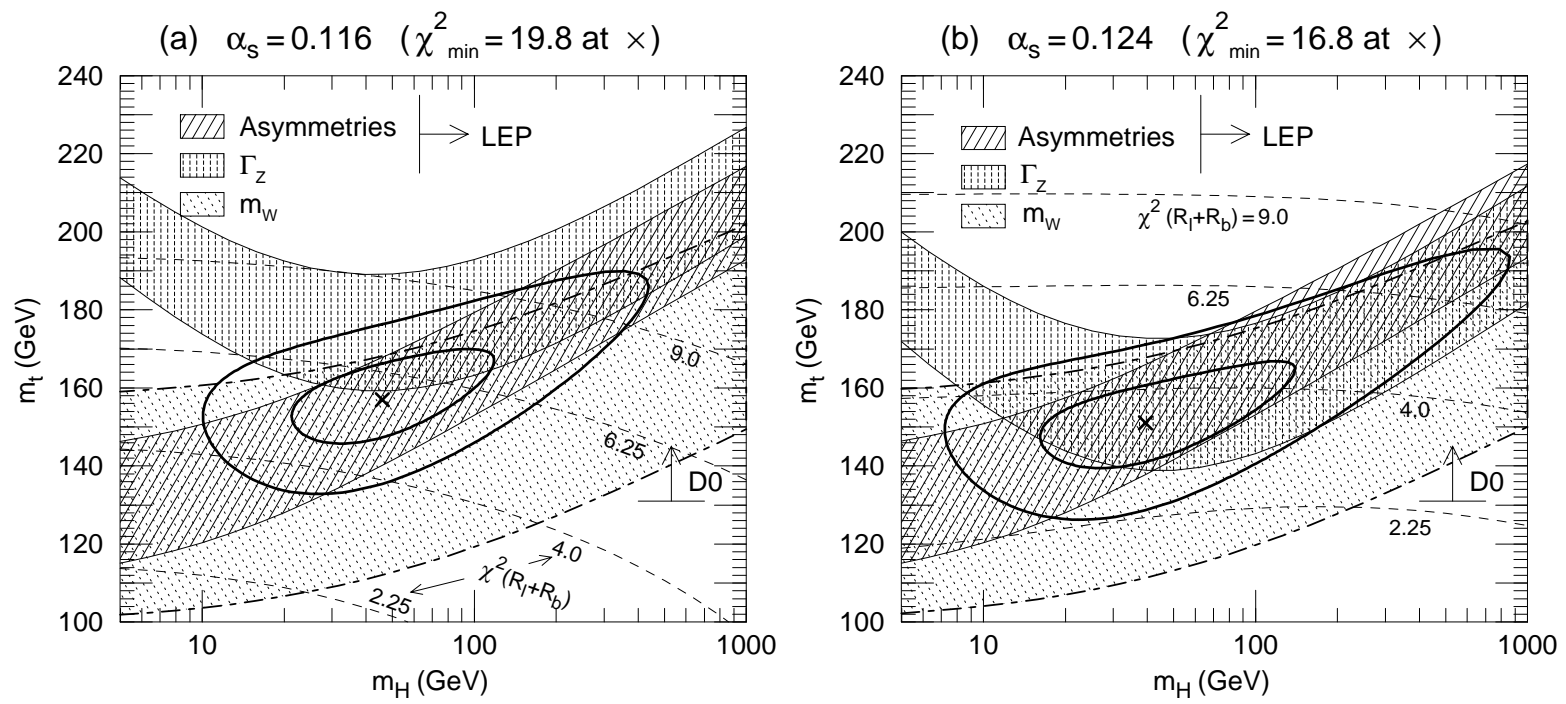

Fig. 3 The SM fit to all electroweak data in the $\left(m_{H}, m_{t}\right)$ plane for (a) $\alpha_{s}=0.116$ and (b) 0.124 , with $\delta_{\alpha}=0$. The thick inner and outer contours correspond to $\Delta \chi^{2}=1(\sim 39 \% \mathrm{CL})$, and $\Delta \chi^{2}=4.61\left(\sim 90 \%\right.$ CL), respectively. The minimum of $\chi^{2}$ is marked by an " $\times$ ". Also shown are the 1- $\sigma$ constraints from the $Z$-pole asymmetries, $\Gamma_{Z}$ and $m_{W}$. The dashed lines show the constraint only from $R_{\ell}$ and $R_{b}$ (see also Fig. 国). They correspond to $\chi^{2}=2.25,4.0,6.25,9.0$. The regions $m_{t}<131 \mathrm{GeV}$ and $m_{H}<63 \mathrm{GeV}$ are excluded by CDF [21] and LEP experiments [19], respectively. 
by :

$$
\chi_{\mathrm{SM}}^{2}\left(m_{t}, m_{H}, \alpha_{s}, \delta_{\alpha}\right)=\left(\frac{m_{t}-\left\langle m_{t}\right\rangle}{\Delta m_{t}}\right)^{2}+\chi_{H}^{2}\left(m_{H}, \alpha_{s}, \delta_{\alpha}\right)
$$

with

$$
\begin{aligned}
& \left\langle m_{t}\right\rangle=164.9+12.5 \ln \frac{m_{H}}{100}+1.0 \ln ^{2} \frac{m_{H}}{100}-2.6\left(\frac{\alpha_{s}-0.116}{0.005}\right)-4.8\left(\frac{\delta_{\alpha}}{0.10}\right), \\
& \Delta m_{t}=9.0-0.07 \ln \frac{m_{H}}{100}-\left(0.24-0.036 \ln \frac{m_{H}}{100}\right) \frac{m_{t}-175}{10}
\end{aligned}
$$

and

$$
\begin{aligned}
\chi_{H}^{2}\left(m_{H}, \alpha_{s}, \delta_{\alpha}\right) & =16.4+\left(\frac{\delta_{\alpha}-0.30}{0.30}\right)^{2}+\left(\frac{\alpha_{s}-0.1240+0.0018 \delta_{\alpha}}{0.0046}\right)^{2} \\
& -\left(\frac{\alpha_{s}-0.1376+0.046 \delta_{\alpha}}{0.0133}\right) \ln \frac{m_{H}}{100}-\left(\frac{\alpha_{s}-0.1347}{0.028}\right) \ln ^{2} \frac{m_{H}}{100}+\left(\frac{\delta_{\alpha}}{0.10}\right)^{2} .
\end{aligned}
$$

Here $m_{t}$ and $m_{H}$ are measured in GeV. This parametrization reproduces the exact $\chi^{2}$ function within a few percent accuracy in the range $100 \mathrm{GeV}<m_{t}<250 \mathrm{GeV}, 60 \mathrm{GeV}<$ $m_{H}<1000 \mathrm{GeV}$ and $0.10<\alpha_{s}\left(m_{Z}\right)<0.13$. The best-fit value of $m_{t}$ for a given set of $m_{H}, \alpha_{s}$ and $\delta_{\alpha}$ is readily obtained from eq. (14b) with its approximate error of (140). For

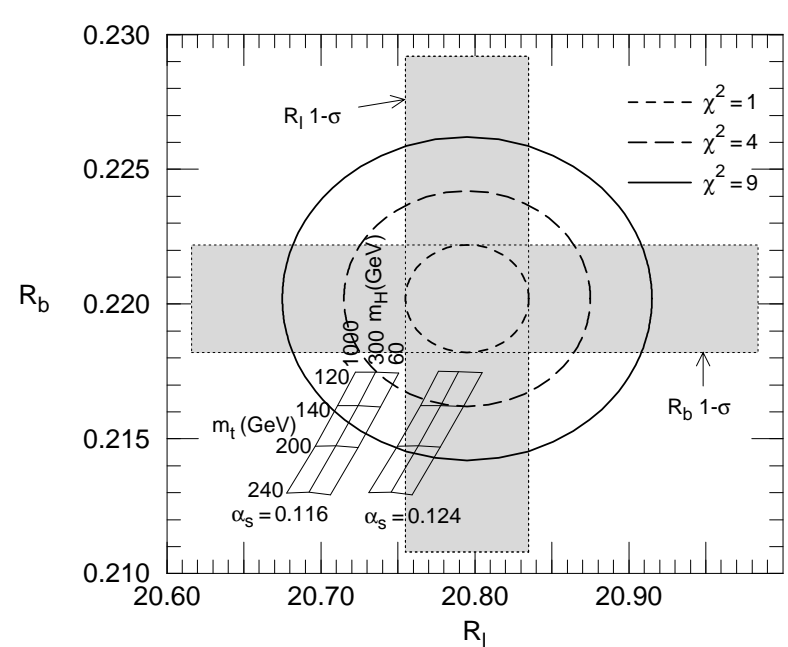

Fig. 4 The $R_{b}$ vs. $R_{\ell}$ plane. The $\mathrm{SM}$ predictions are shown in the range $120 \mathrm{GeV}<m_{t}<240 \mathrm{GeV}$, and $60 \mathrm{GeV}<$ $m_{H}<1000 \mathrm{GeV}$, for two cases of $\alpha_{s}$ $\left(\alpha_{s}=0.116\right.$ and 0.124$)$. Also shown are the $\chi^{2}=1,4,9$ contours obtained by combining the $R_{\ell}$ and $R_{b}$ data.

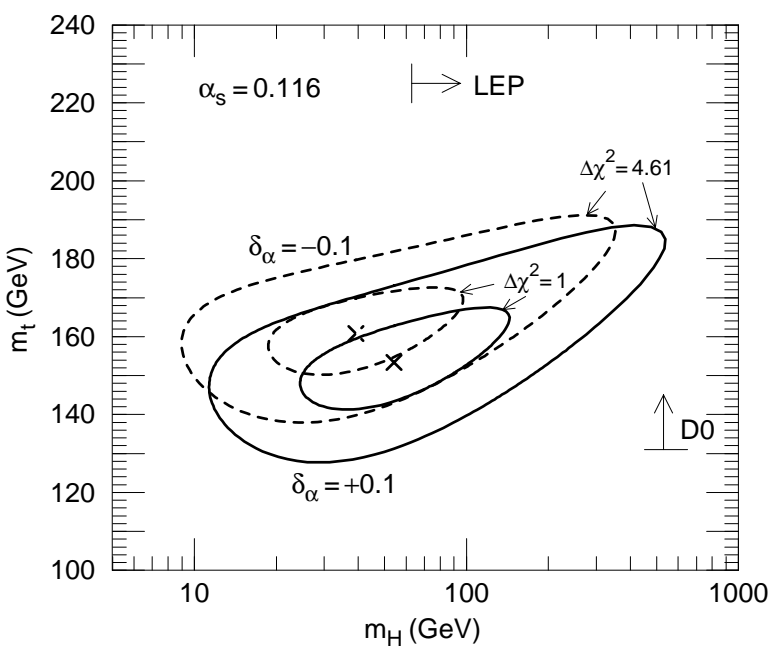

Fig. 5 The SM fit to all electroweak data in the $\left(m_{H}, m_{t}\right)$ plane for $\alpha_{s}=0.116$. The dashed lines show the case for $\delta_{\alpha}=-0.1$, while the solid lines show for $\delta_{\alpha}=+0.1$. The inner and outer contours correspond to $\Delta \chi^{2}=1$ and $\Delta \chi^{2}=4.61$, respectively. 
$m_{H}=60,300,1000 \mathrm{GeV}, \alpha_{s}=0.116$ and $\delta_{\alpha}=0$, one obtains

$$
m_{t}=\left\{\begin{array}{llll}
159 \pm 9 \mathrm{GeV} & \text { for } & m_{H}=60 \mathrm{GeV} & (19.9 / 19) \\
180 \pm 9 \mathrm{GeV} & \text { for } & m_{H}=300 \mathrm{GeV} & (23.1 / 19) \\
199 \pm 8 \mathrm{GeV} & \text { for } & m_{H}=1000 \mathrm{GeV} & (27.8 / 19)
\end{array},\right.
$$

where $\left(\chi_{\min }^{2} /\right.$ d.o.f. $)$ is shown in brackets. We note here that $\chi_{\min }^{2}=27.8$ for $m_{H}=$ $1000 \mathrm{GeV}$ reflects the discrepancies between the fitted value of $m_{t}$ and the $R_{\ell}$ and $R_{b}$ data. One can observe from eq. (14b) that changing $\alpha_{s}$ by \pm 0.005 shifts the best-fit values of $m_{t}$ about $\mp 3 \mathrm{GeV}$, while changing $\delta_{\alpha}$ by \pm 0.1 shifts it about $\mp 5 \mathrm{GeV}$.

Due to its quadratic form it is easy to obtain from eq. (14) results which are independent of $\alpha_{s}$ and/or $\delta_{\alpha}$. Also, additional constraints on the external parameters $\alpha_{s}$ and $\delta_{\alpha}$, such as those from their improved measurements or the constraint from the grand unification of these couplings may be discussed without difficulty.

In view of the recent publication by the CDF collaboration [6] concerning evidence for the top quark with $m_{t}=174 \pm 16 \mathrm{GeV}$, it is instructive to anticipate the impact a precise measurement of the top-quark mass would have in the context of the present electroweak data. In the discussion below we treat $m_{t}$ as an external parameter, and hence we discuss the sensitivity of the present electroweak data to $m_{H}$ while assuming that $m_{t}$

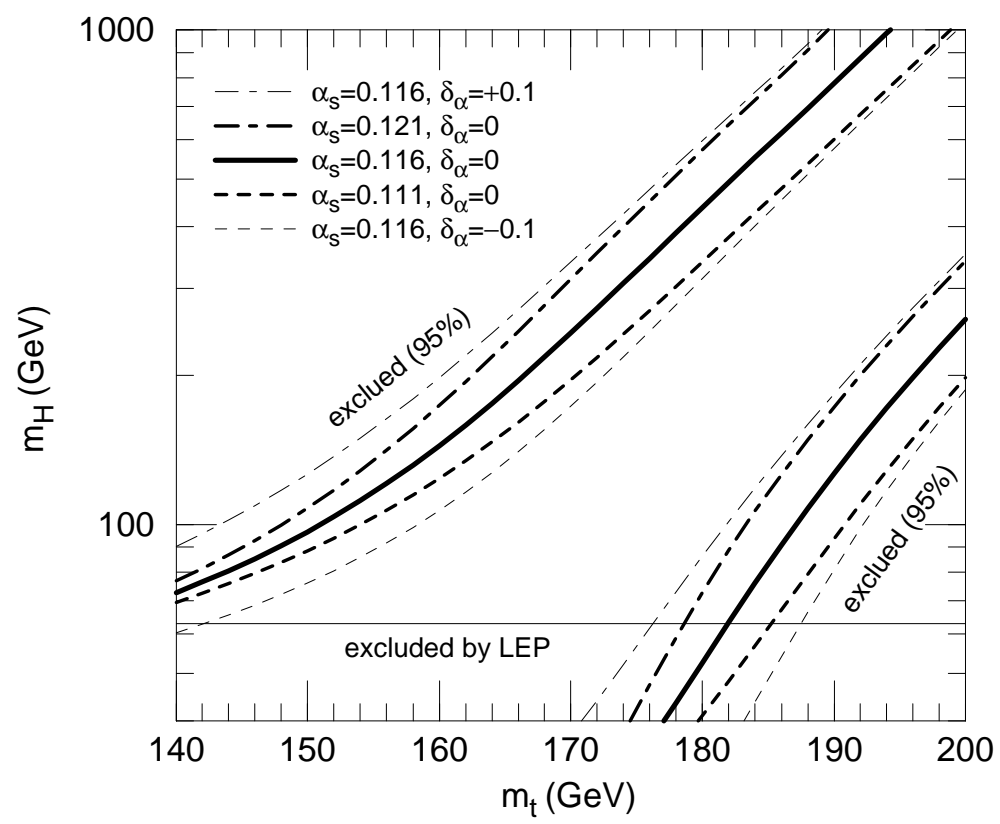

Fig. 6 Constraints on the Higgs mass in the SM from all the electroweak data. Upper and lower bounds of the Higgs mass at 95\% CL are shown as functions of the top mass $m_{t}$, where $m_{t}$ is treated as an external parameter with negligible uncertainty. The thick solid lines show the case for $\alpha_{s}=0.116$ and $\delta_{\alpha} \equiv 1 / \bar{\alpha}\left(m_{Z}^{2}\right)-128.72=0$. The cases for $\alpha_{s}=0.111$ and 0.121 with $\delta_{\alpha}=0$ are shown by thick dashed and dot-dashed lines. The cases for of $\delta_{\alpha}= \pm 0.1$ with $\alpha_{s}=0.116$ are also shown by thin dashed and dot-dashed lines. 
is known precisely. The 95\% CL upper/lower bounds on $m_{H}$ from the electroweak data are shown in Fig. 6 as functions of $m_{t}$. Shown by the thick solid lines are the bounds for $\alpha_{s}\left(m_{Z}\right)=0.116$ and $\delta_{\alpha}=0$. The bounds for $\alpha_{s}\left(m_{Z}\right)=0.111$ and 0.121 with $\delta_{\alpha}=0$ are shown by dashed and dot-dashed thick lines, respectively, while the bounds for $\delta_{\alpha}=-0.1$ and +0.1 with $\alpha_{s}=0.116$ are shown by dashed and dot-dashed thin lines, respectively. In the region $160 \mathrm{GeV}<m_{t}<190 \mathrm{GeV}$ the upper bound on $m_{H}$ at the $95 \% \mathrm{CL}$ is approximately expressed asi

$$
\ln \frac{m_{H}}{100}<\left\{\begin{array}{c}
0.91 \\
1.13 \\
1.38
\end{array}\right\}+\left\{\begin{array}{l}
0.85 \\
0.91 \\
0.95
\end{array}\right\} \frac{m_{t}-174}{16}+0.34 \frac{\delta_{\alpha}}{0.1} \quad \text { for } \quad \alpha_{s}=\left\{\begin{array}{l}
0.111 \\
0.116 \\
0.121
\end{array}\right.
$$

where $m_{t}$ and $m_{H}$ are measured in GeV. For a smaller value of $m_{t}$, a rather stringent upper bound on $m_{H}$ is obtained. Since these bounds are very sensitive to the value of $m_{t}$ as well as the assumed values of $\alpha_{s}$ and $\delta_{\alpha}$ a further, more accurate measurement of $m_{t}$ will give more definite information on $m_{H}$.

To summarize: We have performed a comprehensive analysis of the recent electroweak data at LEP/SLC. The two universal parameters, $\bar{g}_{Z}^{2}\left(m_{Z}^{2}\right), \bar{s}^{2}\left(m_{Z}^{2}\right)$, and the $Z b_{L} b_{L}$ vertex form-factor, $\bar{\delta}_{b}\left(m_{Z}^{2}\right)$, are determined from these data. The $S, T$ and $U$ parameters are also determined by including the data from low-energy neutral-current experiments and $W$ mass data. The errors in $S$ and $T$ are much reduced from those of the previous analysis [7], and simple TC models are clearly disfavored. As for the SM fit, the value of $m_{t}$ favored by the electroweak data is in good agreement with the value favored by CDF [6]. We also note that an upper bound on the Higgs-boson mass can be obtained for a given value of $m_{t}$, and that a stringent upper bound $\left(m_{H}<140 \mathrm{GeV}\right)$ is found for rather small $m_{t}(\lesssim 160 \mathrm{GeV})$. At all stages of our analysis, we have discussed, in detail, the uncertainties coming from the QCD coupling strength, $\alpha_{s}$, and the shift $\delta_{\alpha} \equiv 1 / \bar{\alpha}\left(m_{Z}^{2}\right)-128.72$ by presenting all fit results as functions of $\alpha_{s}$ and $\delta_{\alpha}$. The improvement of the measurements of these parameters are crucial to the search for physics beyond the SM through radiative corrections.

\footnotetext{
†One comment is in order. Although our approximate formulae for the $\chi^{2}$ of the SM fit, (14), reproduce the exact result within about $1 \%$ accuracy in the Higgs-mass range $63 \mathrm{GeV}<m_{H}<1000 \mathrm{GeV}$, one should not use these formulae to find the confidence levels of $m_{H}$ for small $m_{t}$; the neighborhood of the minimum of the $\chi^{2}$ is outside the above range, and in this case the exact $\chi^{2}$ and the approximate formulae are significantly different. See ref. [7] for discussions.
} 


\section{Acknowledgements}

The author wishes to thank B.K. Bullock, K. Hagiwara, D. Haidt, R. Szalapski and Y. Yamada for clarifying discussions. He is also grateful to the KEK theory group for extending its hospitality during the course of this work.

\section{References}

[1] The LEP electroweak working group, LEPEWWG/94-01 (CERN May 1994).

[2] D. Schaile, talk presented at the 27th International Conference on High Energy Physics, Glasgow, Scotland, July 20-27, 1994.

[3] The LEP Collaborations, ALEPH, DELPHI, L3, OPAL and The LEP electroweak working group, CERN/PPE/93-157 (August, 1993).

[4] SLD Collaboration, K. Abe et al., Phys. Rev. Lett. 73, 25 (1994).

[5] CDF Collaboration, talk by K. Hara at 22'nd INS International Symposium on Physics with High Energy Colliders, Tokyo, Japan, Mar. 8-10, 1994 ;

D0 Collaboration, talk by Q. Zhu at 9'th Topical Workshop on Proton-Antiproton Collider Physics, Tsukuba, Japan, Oct. 18-22, 1993.

[6] CDF Collaboration, F. Abe et al., Phys. Rev. Lett. 73, 225 (1994); Preprint FERMILABPUB-94/097-E (April, 1994) .

[7] K. Hagiwara, D. Haidt, C.S. Kim and S. Matsumoto, KEK preprint 93-159, to be published in Z. Phys. C.

[8] M. E. Peskin and T. Takeuchi, Phys. Rev. Lett. 65, 964 (1990); Phys. Rev. D46, 381 (1992)

[9] D.C. Kennedy and B.W. Lynn, Nucl. Phys. B322, 1 (1989).

[10] G. Degrassi and A. Sirlin, Nucl. Phys. B383, 73 (1992); Phys. Rev. D46, 3104 (1992).

[11] G. Degrassi, B.A. Kniehl and A. Sirlin, Phys. Rev. D48, 3963 (1993).

[12] Particle Data Group, L. Montanet et al., Phys. Rev. D50, Vol.3-I (1994).

[13] F. Jegerlehner, as quoted in B.A. Kniehl, Proceedings of the International Europhysics Conference on High Energy Physics, Marseille, France, July 22-28, 1993, ed. by J. Carr and M. Perrottet (Editions Frontières, Gif-sur-Yvette), p. 639.

[14] A. Sirlin, Phys. Rev. D22, 971 (1980).

[15] G.L. Fogli and D. Haidt, Z. Phys. C40, 379 (1988).

[16] G. Rädel and R. Beyer, Mod. Phys. Lett. A8, 1067 (1993).

[17] S.A. Blundell, W.R. Johnson and J. Sapirstein, Phys. Rev. Lett. 65, 1411 (1990). 
[18] C.Y. Prescott et al., Phys. Lett. 84B, 524 (1979).

[19] G. Coignet, talk presented at the XVI International Symposium on Lepton-Photon Interactions, New York, Aug. 10-15,1993.

[20] CDF Collaboration, F. Abe et al., Phys. Rev. D45, 3921 (1992).

[21] D0 Collaborations, S. Abachi et al., Phys. Rev. Lett. 72, 2138 (1994). 